\title{
A novel metric for responding to transport inequality
}

Tom Cohen ${ }^{1}$ - Senior Fellow (Research \& Teaching), Centre for Transport Studies, University College London (UCL), Gower Street, London WC1E 6BT, UK; tom.cohen@ucl.ac.uk; +44 2076792276. orcid.org/0000-0002-3818-2087

John Shrewsbury - Independent Transport \& Traffic Analyst, New Zealand/Australia/UK.

\section{Abstract}

\section{Introduction}

This paper is motivated by the following question: how can someone who wishes to address transport inequality know if they have made progress? On the basis that existing measures do not appear to provide the answer, we sought to develop a new one, drawing on evidence from England's National Travel Survey.

\section{Methods}

We analysed data from the National Travel Survey to obtain a rich picture of the relationship between household income and three facets of personal travel - average speed, absolute cost and relative cost. Our findings provided the justification for developing from first principles a novel measure of an individual's capacity to make desired journeys, based on the time and relative cost involved.

\section{Results}

Analysis of the National Travel Survey confirms that poorer people take longer, on average, to travel a given distance. And, whilst richer people tend to spend more on travel per unit distance, the relative impact of that expense (taking income into account) in fact decreases with wealth.

The metric developed on the basis of these findings, Index of Personal Travel Impact (IPTI), is an estimate at the individual level of the relative impact of desired travel, expressed as an amount per unit distance. It is calculated using the journeys an individual would like to make (as opposed to those they actually make or those that an authority might assume "important") and reflects the specific characteristics of the individual (e.g. car availability) and of the journey (e.g. the need to arrive by a given time). We discuss the feasibility of calculating this index at an individual level, concluding that it is considerably aided by the increasing availability of large data sources and online journey planning tools.

\section{Conclusions}

We conclude that IPTI has potential to inform policy making because it is a meaningful measure and strikes an acceptable balance between accuracy and measurability. Its use of time and relative cost - the two most important resource implications of journey-making for the individual - means that it captures quite well the "headline" impacts of travel whilst being relatively easy to calculate. As defined, it should provide a readily understandable way of seeing the differing extent to which people face mobility barriers. This could be useful in transport planning, especially where efforts are being directed at addressing inequality. By extension, IPTI could also inform an appraisal process

\footnotetext{
${ }^{1}$ Corresponding author
} 
that would demonstrate a given scheme's distributional effects in terms of relative capacity to travel.

The feasibility of collecting the data necessary for IPTI's calculation needs to be investigated as does the scope to use proxies and/or generalisations to make the calculation process more efficient. And future research also could usefully explore the scope for incorporating some externalities associated with travel.

\section{Keywords}

Transport, mobility, justice, fairness, accessibility, measurement

\section{Introduction}

We know that poor people are more dependent on slow and/or indirect forms of transport. In England, for example, people in the poorest income quintile cover six per cent of their overall mileage on foot or by bicycle, compared with two per cent in the highest income quintile, and travel 12 per cent by bus, compared with two per cent in the highest income quintile. In contrast, those in the richest quintile cover 14 per cent of their distance by surface rail whilst the poorest quintile travels six per cent by this mode. ${ }^{2}$ It seems extremely likely that, by implication, it on average takes poorer people longer to travel a given distance, but this has not so far been empirically demonstrated to our knowledge. In this article we provide evidence to support this claim, using data from the UK's National Travel Survey (NTS).

The NTS data also provide us with the opportunity to explore in some detail the real-terms cost of travel per unit distance and how this varies with income. It is well established that poorer people travel less than their richer counterparts and it is asserted that this is attributable in part to the relative cost of travel [2] but how does the true financial impact of travel vary? The NTS data demonstrate that the actual cost of travelling a mile is greater on average for richer people but, when household income is used as a denominator to simulate the financial impact in relative terms, a striking disparity is revealed: a mile's travel affects the poorest twentieth more than twice as much as it does the next twentieth and more than ten times as much as the richest twentieth.

These findings accentuate the need to capture, as a minimum, time and relative financial impact if attempting to gauge the individual effect of travel to inform the policy development process, especially where the aim of any policy is to address transport inequality. Whilst we cannot in this paper include a formal survey of such metrics, we note that many accessibility measures fall short of this requirement because they are based on travel time and omit money [3]. Many others are so complex as to be unusable [4]. Responding to these findings, we propose a novel metric that satisfies the two requirements set out above whilst also being simple enough to make calculation ostensibly feasible. In this paper, we set out the formulation of the metric which we call the index of personal travel impact (IPTI), discuss a number of aspects of the formulation and assess its potential use in the policy-making process.

\subsection{Background}

There is a growing literature concerning the relationships between personal accessibility and mobility, individual wealth and the more recent concepts of social exclusion [5] and "transport poverty" [e.g. 2, 6]. This reflects an increasing awareness and acceptance that there are strong, if complex, connections between the extent to which people can and do travel and other important

2 Derived from [1]; data for 2015. 
aspects of their well-being. And this has informed a further flowering of research in the field of transport or mobility justice $[7,8]$. More specifically, the evidence that transport "injustice" may beget or exacerbate more general forms of social injustice has stimulated various transport and mobility researchers to investigate what policies are needed to foster a more just transport system, or one that is likely to promote wider justice (this being a distinct goal).

The dominant set of measures used so far in this debate are those of accessibility (rather than mobility). Accessibility, broadly defined, is the ease with which individuals are able to reach important destinations and/or obtain the things that they need or desire in order to have a fulfilling life. As a consequence, accessibility measures can be expected to address personal circumstances, locations of services and activities, and the nature of the transport network and system [4].

The very large number of factors in the above list of accessibility "components" helps to explain the diversity of accessibility measures [9] and the fact that the majority of measures in use omit some components and/or simplify others. This, and the fact that accessibility is a relatively new concept when compared with many other aspects of transport research, help to explain the continuing innovation in the field, with researchers critiquing the state of the art and offering new methods that they argue will improve upon current practice [e.g. 10, 11].

The critiques of accessibility measures include that they often exclude the financial cost of travel, concentrating on the time involved [3] and that they do not readily enable the quantification of benefits arising from transport interventions [12]. In fact, Curl et al. [12] attribute the relative lack of policy impact achieved by accessibility measures to this deficiency. As Lucas [13] puts it, there is considerable room for, and need for, improvement.

Not that accessibility measures are alone in being considered to fall short of the ideals set for them. The relative simplicity of basing analysis on the journeys that people make risks missing the highly significant point that the "transport poor" may be forgoing journeys that they wish or need to make, because of the cost of time involved. To give just one example of this, Moore et al. [14] find evidence that journeys to visit friends and relatives are suppressed amongst those on low incomes. Not only does this illustrate elegantly the problem with mobility-based measures, it also helps to show that accessibility measures based only on "essential" trips (such as journeys for work, health, education and grocery shopping) can miss less obvious travel that can make a major contribution to well-being, as discussed below.

\subsection{Moving beyond accessibility}

We have already asserted that the omission of financial impact from many accessibility measures is a significant weakness. Another weakness is the very considerable quantity of data required in order to estimate accessibility at a spatially specific level. The advent of "big data" as well as sophisticated means of analysing data sources are beginning to lessen the significance of this issue but it remains. For example, whether a given bus route is a feasible means for Person A to commute to work depends not just on where it runs and its frequency, but whether the first and last bus times are consistent with her shift pattern and whether she is even able to use it. That is, true accessibility is a function both of the nature of the transport network and a complex set of personal circumstances [4].

We argue that a very significant additional weakness lies at the conceptual, rather than practical, level. It is that measures of accessibility typically depend on assumptions concerning which destinations are important or essential. To give a prominent example from the UK, the practice of accessibility planning was based on a set of general indicators based on journey times to given types 
of destination. The destinations are primary and secondary schools, further education establishments, "work", hospital, GP and major centres [15]. At face value, this is reasonable: health, work, education and the opportunities available at a major centre all appear high priorities for individual travel. But it is easy to imagine an example that shows the limitations of this method. A person may live in the centre of a major city and have easy access to all of these essentials but at the same time be isolated from their loved ones who are in a neighbouring city, joined by a rail link that is exorbitantly expensive.

Such measures are unavoidably paternalistic because they impose on individuals a set of assumptions concerning where they would need or want to go. And, because the set of assumptions is homogeneous, it also glosses over individual specificities: if I have a very particular skill, I may not be able find employment in the local town, whether or not there is a bus service to it. The blanket approach also necessarily misses the very significant matter of other people whom I may want to see. Accessibility measures that do not draw on the individual's preferences are not likely to be able to extend to this level of detail.

A lesser concern relates to aggregation. Where accessibility data have been gathered for a range of "desirable" destinations, can these be readily combined into a single figure [12]? We have already identified the difficulty with having to make assumptions on behalf of others. Even once blanket assumptions have been made, how should we "sum" the accessibility of the nearest hospital with the accessibility of the nearest major centre? Some weighting (implicit or explicit) is necessary if a set of values is to be condensed into a headline figure and this will have to be done at the aggregate level, meaning that differences between individuals will need to be averaged out.

This is not to disparage accessibility measurement as a whole. Accessibility measurement can be immensely helpful in a specific planning context, when deciding on the location of new services, for example. It can also usefully inform the modification of transport services in terms of whether a change would be an improvement on the status quo. We argue, though, that the policy maker seeking to tackle transport inequality requires a more nuanced tool, as explained in subsequent sections.

\subsection{Structure of the paper}

The paper is structured as follows.

Section 2 sets out the analysis we conducted using NTS data which provides the basis for our claim that time and relative cost should feature in any measure designed to support the addressing of transport inequality;

Section 3 introduces our Index of Personal Travel Impact, explaining and discussing its formulation;

Section 4 addresses the feasibility of making the Index operational and discusses ways in which it could be further developed; and

The conclusion (Section 5) discusses the possible use of the Index to support policy making.

\section{Using the NTS to understand the relationship between wealth and impact of travel}

In this section, we describe work done to investigate the relationship between wealth and the impact of personal travel, using data from England. The focus of the description is the method used 
and results obtained; further information concerning the NTS and decisions we made when carrying out the analysis is provided in the appendix.

\subsection{Method}

We used a dataset from the National Travel Survey (NTS) supplied to us by the UK Data Archive [16]. The NTS is a long-established rolling survey based on large samples, now covering England (rather than the whole of Great Britain). In addition to collecting a large quantity about the characteristics of individuals and households, it gathers a week's detailed journey-making information from all members of participating households. The dataset supplied to us is an anonymised collation of cleaned data collected over the period 2002 to 2014.

For the analysis we wished to carry out, the key data we needed on trips made related to mode, distance, time and cost. For journeys made by private motorised modes, we needed distance and time (and we imputed operating cost, using a flat quantity per $\mathrm{km}$ ). For public transport modes, we additionally needed fare paid.

To enable the analysis we wished to carry out, we aggregated data on journeys made to the household level because this is the unit at which income is recorded.

\subsection{Findings}

Two central findings result from our analysis, relating to a) speed and b) cost of travel, with respect to household income.

\subsubsection{Speed}

As Figure 1 shows, there is a clear relationship between income and the time taken to travel a given distance, in this case a mile. The correlation is very nearly monotonic, with only a slight increase in time between the poorest $5 \%$ and the next semi-decile (twentieth). And the difference is quite stark, with the "slowest" category taking on average nearly 4.5 minutes to travel a mile, compared with 2.4 minutes for the wealthiest category.

Fig. 1: Mean travel time per mile by income band

On reflection, this is hardly surprising. We know that poorer people use buses to a greater extent than richer people [1] and this is very likely to mean slower journeys in almost all cases than the equivalent by car: the typical car trip starts nearer the origin and finishes nearer the destination, travelling very often by the shortest path and quite possibly making no intermediate stops. In addition, walking and cycling both account for a larger proportion of overall distance travelled by people in lower-income groups and, except in conditions of heavy traffic or high levels of traffic management (a lack of through routes etc), they are slower than private motorised modes. In contrast, surface rail, typically quick, represents a much larger proportion of the distance travelled by people in the highest-income group than in the lowest-income group.

\subsubsection{Cost}

The absolute cost of travel per mile by income band is shown in Figure 2 . This reveals a slightly more complex relationship than in the case of speed. Here, the lowest cost per mile is borne by the fourth income band, with both those in lower- and higher-income groups paying more for travel. This relationship is probably explained by two factors operating together: the increasing role of private car as income rises (typically cheaper per unit distance than public transport), and the increasing preference for (comparatively expensive) rail amongst the highest-income groups. The lowest point in the graph may represent an intercept between reducing bus use and increasing rail use. 
Fig. 2: Mean cost of travel per mile by income band

A more interesting (and consistent) picture is presented in Figure 3. This shows the result of dividing cost per mile by household income for each of the bands (indexed to the poorest five per cent), thereby showing the approximate true financial impact of travel. There is one "kink", with the $16^{\text {th }}$ band having a slightly higher value than the $15^{\text {th }}$, but the pattern is otherwise uniform and very clear: the real financial impact of travel is over twice as great for the poorest five per cent than for the next five per cent. And the impact felt by the wealthiest semi-decile is less than a tenth of that of the poorest. What this demonstrates very effectively is that any positive correlation between wealth and cost per mile (as shown in Figure 2) is more than outweighed by income effects: wealthy people may choose slightly more expensive ways of travelling but they still feel less of an impact per mile.

Fig. 3: Mean cost of travel per mile as proportion of household income, by income band

\subsection{Implications}

These findings serve to reinforce what has already been known for some time: that the richer you are, the more mobile this makes you. But, though we already knew that richer people travelled further than poorer people, this analysis helps to confirm that capacity to travel is closely related to income too. This is important in policy terms because capacity to travel is strongly correlated with the opportunities that are available at the end of a journey. More specifically, if valuable destinations are evenly distributed and travel is possible in all directions, the number that can be reached will be proportional to the square of one's speed of travel. This relationship further accentuates the disparity already demonstrated with respect to income and speed.

What is true of speed is equally true of cost: the less financial impact travel has upon you, the further you can travel and the greater the number of opportunities therefore available to you. And opportunities can be interpreted in at least two ways: in a basic economic sense, an opportunity may be a place where it is possible to find work. Up to a point, the more job opportunities, the greater chance there is of finding satisfactory work. The second interpretation is based more on well-being: again, up to a point, the more destinations available to one, the richer life can be. There are limits in both cases - one needs only so many job offers, bingo halls or concert venues - but, until such a limit is reached, the benefits of greater access are real.

Up to this point, personal wealth has for good reason been our reference point: it has enabled us to enhance our picture of disparities amongst English people in terms of their journey making. But we need now to move on to a broader interpretation of travel impact in order to reflect the fact that it is possible to be moderately wealthy but face transport barriers nonetheless. Moreover, we need to go beyond an analysis based only on the journeys people do make as opposed to those they would like to be able to make.

Therefore, in the second part of this paper, we use the components of cost and speed to develop a measure of personal travel impact that takes account of disposable income as well as travel aspirations.

\section{Constructing a measure of personal travel impact}

When policy makers seek to tackle transport inequality, a problem they immediately encounter is the choice of metric. As we have discussed and demonstrated above, transport inequality is widespread and, in some cases, extreme. But there is no one measure that has been generally agreed upon as a suitable reference point. In other words, if a policy maker sets out to address transport injustice, how will she know whether she has succeeded? 
Before we proceed, we should define the term "impact" as we are using it here. By personal travel impact, we are talking about the consumption at the personal level of finite resources by the act of travelling. As we go on to explain, the resources on which we concentrate are money and time though impact could extend to physical, cognitive and affective energy, and so on.

\subsection{A measure based on money and time}

Taking the complexity of accessibility measurement as a useful reminder, we seek a metric which strikes a successful balance between precision and ease of application. The more data that are included, the more accurate we might expect the metric to be, but the effort required to develop and implement any metric will be proportional to its complexity.

We start with generalised cost, which has long been used to support forecasting and appraisal, though its popularity is not universal [17]. This quantity can take a range of forms but, at its most basic, it is a linear combination of the cost and time associated with travel [18]. More sophisticated versions of generalised cost differentiate between time spent in a vehicle and time travelling to or waiting for it; they may also include quantities such as an "interchange penalty" to reflect the fact that people generally prefer direct journeys if they are available. ${ }^{3}$ But the simplest formulation reflects the fact that the dominant elements of the personal impact of travel are the time it takes and the money it costs. Though of course a simplification, this version of generalised cost captures the reality of travel reasonably well. The fact that researchers have attempted to express changes in accessibility through generalised cost [20] suggests that others see its potential usefulness when investigating the social impact of transport.

One area in which we would part ways with conventional versions of generalised cost is its treatment of money. The absolute cost of travel is used in formulations of generalised cost and this reflects its roots in microeconomics. From a policy perspective, we argue that the real impact of transport needs to be shown and this is a function of disposable income, for which total gross income is the best available proxy, as used above in our NTS analysis. We therefore estimate the real financial impact of travel by calculating the ratio of the financial cost of travel to the person's estimated total income.

Because we have adopted a deliberately simple formulation of generalised cost, we require a single measure of journey time. We select door-to-door journey time as this seems the best way to capture the different and often overlooked aspects of travel such as walk access/egress and parking search. This glosses over the fact that travellers dislike certain aspects of travel (such as waiting for a vehicle) more than others, which more sophisticated formulations of generalised cost reflect through weighting but, again, this omission is justified by the need to limit the complexity of the measure.

\subsection{Combining money and time}

The convention in estimating generalised cost is to convert the constituent elements into a single unit of account. Those elements that are initially measured in units of time are translated into monetary values using the so-called "value of time". This quantity has been extensively researched [e.g. 21] and continues to be debated [e.g. 22] but it has become more or less an accepted part of transport planning.

3 In fact, Transport for London [19] has one of the most sophisticated sets of journey parameters, including the value placed by customers on the absence of graffiti. The quantities are, however, small in comparison with travel time and cost. 
In our case, because we are adjusting financial impact to reflect personal income, we do as follows.

The raw financial impact of a journey is income-adjusted by multiplying it by the average personal income of the area of interest (perhaps an entire country or a region within it) then dividing by the individual's personal income. Thus a journey costing $f 10$ in "raw" terms has an adjusted cost of $\mathrm{f} 12.50$ when made by someone with a personal income that is $80 \%$ of the average.

Having done this, we use the prevailing average value of time as estimated for the community in question in order to bring the time and money elements into the same unit of account.

To continue the example above, let us assume that the adjusted cost of a trip is $f 12.50$ and that it would take 40 minutes, door to door (including any time for access, egress, walking, waiting, parking search etc). Supposing the current value of time is $£ 0.10$ per minute, we can convert the adjusted cost of the trip into time by dividing by the value of time to give 125 minutes. We sum this and the actual journey time to produce a total of 165 minutes. ${ }^{4}$

\subsection{Deriving a value at the person level}

It is now necessary to move from the impact of a single journey to an estimate of the impact at the level of the individual. This requires a suitable number of representative journeys to avoid the estimate being skewed by one or two journeys that have disproportionately low or high impact. Whether this is 10, 15 or 50 journeys is a matter of trading off the additional calculation burden against the additional accuracy it would bring. The representative set should reflect not just the range of journeys that the individual wants to make but their likely frequency - weighting could be used to include frequency in order to keep the calculation manageable.

It is important is to assert at this point that the journeys used should be those an individual wants to make, whether or not they are currently realistic (given the individual's circumstances and the nature of the transport network). This is crucial in order for the metric to avoid being determined by an individual's currently constrained travel habits. Instead, the use of journey aspirations ensures that the metric for personal travel impact correctly gauges the match between the individual's ability to travel and the destinations that are important to her.

For each of the journeys in the set, the time and financial impacts need to be estimated. These will reflect the individual's circumstances: if she has access to a car and is able to drive, this is treated as a feasible mode; if she must arrive at her destination by 5 am and does not have access to a car, this may mean that the journey has to be made by taxi/private hire; if she is a wheelchair user, this will be reflected in the set of feasible options. Ordinarily, the estimate should be based on the lowestimpact feasible option, where "impact" is the combination of time and (income-adjusted) financial cost as described above.

The income-adjusted financial impacts and total journey time of a set of representative desired journeys are combined into a single total. Then the final stage in moving towards an index at the individual level is to divide this total by the combined distance of the set of journeys, thus producing an impact per unit distance. The measure used is crow-flies distance across the set of journeys. We call the resulting ratio the Index of Personal Travel Impact, or IPTI.

We present an expression for this quantity $(I)$ below:

4 In this instance, the common unit chosen is minutes; journey time could equally be multiplied by value of time in order to arrive at a combined quantity expressed in money terms but the effect would be the same. 


$$
I=\frac{\sum_{j} t_{j}+\frac{N}{V . i} \sum_{j} c_{j}}{\sum_{j} d_{j}}
$$

Where:

$t_{j}$ is the door-to-door journey time of the lowest-impact option for trip $j$

$c_{j}$ is the absolute financial cost of the lowest-impact option for trip $j$ in terms of fares paid, vehicle running costs etc ${ }^{5}$

$N$ is the average personal income of the area under consideration

$V$ is the prevailing value of time

$i$ is the individual's income

$d_{j}$ is the crow-flies distance between the origin and destination of trip $j$

\subsection{Discussion}

Various aspects of the formulation of IPTI deserve a degree of examination, as follows.

\subsubsection{The role of time in IPT|}

The somewhat trite assertion that we all have 24 hours per day is nonetheless an accurate one. This can be countered with claims that some people are relatively "time-rich" or "time-poor", reflecting the commitments in their lives. Such claims of course have some foundation and a case could be made for calculating the time taken by a person to travel a unit distance relative to their "time budget". If Person A (working full-time with two young children) has one hour available (not already committed to another activity) per day whereas Person B (retired) has four, this would suggest that ten minutes' travel would have four times the impact for A as for B. Leaving aside the very considerable data requirements associated with adding this dimension into the analysis, we demur on the grounds that choice plays a significant part in the structure of our lives. Being time-rich or time-poor can of course be greatly affected by factors beyond our control (the need to care for a sick relative, say) but it is also largely a product of decisions made autonomously. If I choose to volunteer with three charities as well as working full-time, I can expect to be busier than my neighbour, also employed full-time but with no volunteering commitments. Given the motivation for developing the Index of Personal Travel Impact - addressing inequalities in the capacity to reach destinations of importance - it is far from obvious that the person undertaking more activities should expect to enjoy a higher quality of transport options, as would be implied if the metric were based on "disposable time".

\subsubsection{Dividing by distance}

Why create an "index" rather than simply use the sum of impacts across the representative journeys selected by the individual? This is best demonstrated by picturing an individual whose selected journey aspirations include a trip to the Caribbean. First, we must emphasise that the method we set out in this paper explicitly does not question or criticise such a choice - for some people, periodic trips to exotic locations are highly prized. But it must immediately be acknowledged that the personal impact of travelling to such destinations (reflecting journey time and the cost of flying to such destinations) would probably make such individuals' "impact total” considerably higher than

5 The estimation of IPTI could be rendered more accurate by including addition cost elements but at the expense of simplicity. 
those of people whose travel aspirations did not involve international aviation. Remembering, as with the discussion of time above, that the motivation for creating this measure is to address inequalities in ability to travel, it would seem perverse to treat someone who desired long-distance travel as intrinsically deserving of assistance, ceteris paribus. There is the additional risk that, if a sum of the sort discussed were used, individuals would be inclined to "game" the system when listing their desired journeys, a point revisited below in discussion of making the system operational.

A more minor, instrumental benefit of creating a ratio is that it would remove the need to stipulate the number of journeys on which an individual's "score" might be based. If Person A's index is based on 10 journeys and that of Person B based on 50, we would expect the value for Person B to be more accurate but the two quantities should nonetheless be broadly comparable.

It seems natural to use distance as the denominator since we travel in order to cross territory and there is a natural logic to the idea that, the further we go, the greater the impact will be, on average. But its use has an important consequence which should not be overlooked: by expressing personal travel impact as a quantity per unit distance, we exclude the issues of development density and locations of services. Two people may have the same IPTI but one's GP may be less than a mile from her home whilst another must travel ten times that distance.

We have set out above an argument for moving away from conventional accessibility measures and here add that locations of services, though connected with the ability to move, are a distinct issue. And it is easy to see that a goal of equalising the travel impact of reaching one's GP, say, would be likely to produce unwelcome consequences: at the extreme, it may require the provision of supersonic transport to those living in the most remote areas. Not only would this be unsustainable in a variety of ways, it would also provide a perverse incentive to those contemplating a move to a location of low density, with sprawl a likely result. The use of a distance-based denominator reflects the natural travel advantages enjoyed by those living in densely developed locations.

Moreover, it should not fall to the transport sector to compensate for deficiencies in the planning of health, education etc, nor to the idiosyncrasies of a market-based system that may create food deserts through the closure of life-line shops. In fact, the concept of accessibility management [15] was founded on an acknowledgement that transport services should be planned in collaboration with those of the health, education and other sectors, with the explicit aim of avoiding anomalous situations in which services were located inconveniently and transport services expected to provide a solution.

\subsubsection{Crow-flies distance as the denominator}

Having argued for the use of distance as the basis for deriving the IPTI ratio, we must now justify using crow-flies distance rather than the perhaps more familiar choice of distance travelled (as used in the National Travel Survey). Here, whereas we argue that it should not fall to the transport sector to compensate for the peculiarities of where services are located, we claim that it very definitely does behove the transport sector to respond to the fact that some journeys are considerably more circuitous than others. The use of crow-flies distance will ensure that, the more a journey departs from a straight line between origin and destination, the higher its contribution to an individual's IPTI. In some cases, this will reflect topography - island-dwellers who depend on the mainland for key services will be greatly affected by the directness of any links between the two - but it will also frequently be a function of the differing densities of the various modes' networks. For example, the rail network in the UK is for the most part much sparser than its highway counterpart. Journeys by rail will therefore in general involve greater distance, especially as the traveller is not at liberty to charter a train to follow the shortest path along the line but must instead accept the train services 
available, which may mean travelling into and out of a centre in the absence of orbital services. Thus, those who rely on public transport will typically exceed the straight-line distance between origin and destination by a higher proportion than those who have private transport, all other things being equal, and this will (rightly) be reflected in a higher IPTI.

Those who have the use of private transport may equally be forced to divert considerably from the straight-line route in locations where the highway network is itself relatively sparse. Again, this will (properly) be reflected in a higher IPTI for people in these areas compared with those living in areas with denser highway networks.

\section{Next steps}

Having defined IPTI, it is necessary next to investigate the practicality of making it operational. In particular, how much information needs to be collected in order for an individual's value to be calculated with sufficient accuracy, and how much can be estimated reliably using proxies? As more and more data concerning travel behaviour enter the public domain and journey planning becomes increasingly easy using online tools, the burden should be considerably less than would have been the case a decade ago. But there is a presumption that the individual will need to be involved in the calculation process: only they know exactly which journeys they would like to be able to make, and it is probably necessary for now to rely on them to say which travel options are actually feasible.

This leads to questions about resources required and motivation of respondents: why should I take the trouble to provide this information? In the short term, it will be possible to calculate IPTI for small samples of individuals on a market-research basis, compensating respondents for their time. At such time as IPTI became part of the policy-making process, we would need to have information for much larger groups, making payment for the information unworkable. The solution may lie in the prospect of IPTI acting as a mechanism for people to obtain assistance with their travel. Those whose IPTI is likely to be high (because of low income, lack of access to private transport, disability and/or remote location) could be invited to provide this information in the form of an "application for assistance".

As mentioned above, an information gathering process based on self-completion would be at risk of being "gamed" by individuals seeking to increase their IPTI in order to receive benefit. There would therefore need to be certain safeguards in place to manage this risk and future research would need to investigate and appraise the options.

A separate consideration is whether there is scope for any aggregation. At first sight, this is not possible given what has been said about allowing for each individual's particular circumstances. But, as a dataset began to emerge, it may be possible to discern patterns amongst the responses such that it became possible to estimate IPTI using a subset of the information required at first. Certain information, such as the set of public transport options, will be common to individuals across a number of households, for example. A filtering process could then be applied to establish whether a specific person could meet a given journey requirement using available services.

Rather different is the question of externalities. As currently defined, IPTI does not account for any impacts that may fall on someone other than the individual; it does not privilege sustainable transport modes, for example. Future development of the concept could include an investigation of the feasibility and desirability of changing this. 


\section{Conclusion: the potential use of IPTI}

In this paper we have provided new insights into the differing impact of personal travel across members of the population. This has led into the definition of IPTI, intended to be a meaningful measure of individuals' freedom to "get where they want to go". As explained, the formulation has been designed to strike a balance between accuracy and resource requirement (in terms of data collection and analysis). In this regard, its relative simplicity may be a benefit in terms of making it intelligible to the layperson.

IPTI may prove useful to policy makers who are interested in questions of transport and mobility justice. It should provide a readily understandable way of seeing the differing extent to which people face mobility barriers. And, in terms of transport planning, it should enable useful analysis of the spatial distribution of people who face the greatest obstacles to travel. Combined with a method of application, it could inform a fresh approach to transport planning. This will be the subject of a second paper.

\section{References}

1. Department for Transport (2016) Travel by household income quintile and main mode / stage mode: England, 2015 (Table NTS0705)

2. Titheridge H, Christie N, Mackett R, et al (2014) Transport and Poverty A review of the evidence

3. Litman T (2016) Transportation Affordability. Evaluation and Improvement Strategies. Victoria Transport Policy Institute, State of Victoria, Australia

4. Geurs KT, van Wee B (2004) Accessibility evaluation of land-use and transport strategies: review and research directions. J Transp Geogr 12:127-140 . doi:

10.1016/j.jtrangeo.2003.10.005

5. Social Exclusion Unit (2003) Making the Connections: Final Report on Transport and Social Exclusion. Social Exclusion Unit, London

6. Bourn R (2012) Transport and Poverty: A Literature Review. Campaign for Better Transport, London

7. Martens K (2016) Transport Justice: Designing fair transportation systems, 1 edition. Routledge, New York, NY

8. van Wee B (2011) Transport and ethics: ethics and the evaluation of transport policies and projects. Edward Elgar, Cheltenham, UK ; Northampton, MA

9. Papa E, Silva C, Brömmelstroet M te, Hull A (2015) Accessibility instruments for planning practice: a review of European experiences. J Transp Land Use 9: . doi: 10.5198/jtlu.2015.585

10. Preston J, Rajé $F$ (2007) Accessibility, mobility and transport-related social exclusion. J Transp Geogr 15:151-160 . doi: 10.1016/j.jtrangeo.2006.05.002

11. El-Geneidy A, Levinson D, Diab E, et al (2016) The cost of equity: Assessing transit accessibility and social disparity using total travel cost. Transp Res Part Policy Pract 91:302-316 . doi: 10.1016/j.tra.2016.07.003 
12. Curl A, Nelson JD, Anable J (2011) Does Accessibility Planning address what matters? A review of current practice and practitioner perspectives. Res Transp Bus Manag 2:3-11 . doi:

10.1016/j.rtbm.2011.07.001

13. Lucas K (2012) Transport and social exclusion: Where are we now? Transp Policy 20:105-113 . doi: 10.1016/j.tranpol.2012.01.013

14. Moore J, Lucas K, Bates J (2013) Social disadvantage and transport in the UK: a trip-based approach. University of Oxford Transport Studies Unit, Oxford

15. Department for Transport (2006) Accessibility Planning Guidance: Full Guidance. Department for Transport, London

16. Department for Transport (2016) National Travel Survey, 2002-2014: Special Licence Access. [data collection]. 4th Edition. UK Data Service. SN: 7553

17. Grey A (1978) The generalised cost dilemma. Transportation 7:261-280 . doi: 10.1007/BF00165495

18. Searle G (1978) Generalised cost: Fool's gold or useful currency? Transportation 7:297-299 . doi: $10.1007 / B F 00165498$

19. Transport for London (2013) Business case development manual

20. Koopmans C, Groot W, Warffemius P, et al (2013) Measuring generalised transport costs as an indicator of accessibility changes over time. Transp Policy 29:154-159 . doi: 10.1016/j.tranpol.2013.05.005

21. Wardman M, Chintakayala VPK, de Jong G (2016) Values of travel time in Europe: Review and meta-analysis. Transp Res Part Policy Pract 94:93-111 . doi: 10.1016/j.tra.2016.08.019

22. Lin W (2012) Wasting Time? The Differentiation of Travel Time in Urban Transport. Environ Plan A 44:2477-2492 . doi: 10.1068/a4525

23. Morris S, Humphrey A, Byron C, et al (2015) National Travel Survey 2014. Technical Report. National Centre for Social Research, London

24. Department for Transport (2015) National Travel Survey 2014: Notes and Definitions. Department for Transport, London

25. Department for Transport (2014) National Travel Survey. Data Extract User Guide, 1995-2014. Department for Transport, London

26. Department for Transport (2016) Road traffic (vehicle miles) by vehicle type in Great Britain, annual from 1949 (Table TRA0101)

27. Beige S, Axhausen KW (2006) Residence locations and mobility tool ownership during the life course: results from a retrospective survey in Switzerland

28. Department for Transport (2016) Long distance trips within Great Britain by main mode and length: England, 2011/15 (Table NTS0317) 
29. Department for Transport (2016) Sample numbers on which analyses are based: England, 1995 to 2015

30. Department for Transport (2016) Average trip length by main mode: England, 1995/97 to 2015 (Table NTS0306)

\section{Appendix - the National Travel Survey and our use of it}

\section{The National Travel Survey}

The National Travel Survey (NTS) first took place in Great Britain in 1965 and was repeated sporadically until 1988, when it began to be carried out on a continuous basis. In 2002, its sample size increased to over 15,000 households. It has undergone numerous changes since ${ }^{6}$ but it continues to support analysis of trends in travel behaviour in England [23]. Error! Reference source not found.

The NTS uses two data collection methods: face-to-face interviewing using computer-assisted personal interviewing (CAPI) and self-completion of a seven-day travel record. The Household questionnaire provides household-level information about income. It is completed face-to-face with the Household Reference Person (HRP), being the householder with the highest income, or their spouse or partner. Amongst other things, the HRP is asked a question about the household's total gross income which is answered using a show-card. ${ }^{7}$ This places the respondent's household income in one of 20 bands. The McClements scale is subsequently used to adjust the raw answer to reflect household structure: the income of a two-adult household without children is not weighted; the incomes of households with more occupants are scaled down and those of households with fewer are scaled up to create an estimate of "real household income equivalent". Answers are then deflated to 1990 values for comparison purposes [24] which results in a degree of re-banding.

All individuals within a surveyed household are asked to complete the travel record (diary). For each trip made, they are asked to record a range of information, including purpose, origin and destination, departure and arrival times. For multi-stage trips, details for each stage are requested. Long trips (of 50 miles or more) are recorded over two weeks and are logged separately from shorter trips.

For public transport cost, we selected the variable "Stage Cost" as the best available proxy as it is a sum of the responses for single and return tickets, one-day travelcards and pre-pay costs. Of the various samples within the dataset, we used the "diary sample" because this matches best with those who completed the travel record (diary). With respect to household income, the variable we used for our analysis is "Household Income Semi-deciles diary sample".

Of the 103,222 household records in the original set, those that had reported no travel were omitted, leaving 100,684. In doing our work, we complied with the guidance provided with the NTS data concerning use of a series of sample weights [25].

6 The most notable of these is the omission, since 2013, of Scotland and Wales from the sample. The sampling level for England has remained the same as before, enabling longitudinal comparisons for England to continue to be made.

7 Income data are not collected at the individual level in the NTS. 


\section{Limitations of analysis}

The NTS dataset is both very large and quite complex. In order to have a manageable analysis task, we were obliged to omit some detail and to make a number of blanket assumptions, as follows:

- We did not attempt to capture the effective cost of public-transport trips made using season tickets. Survey respondents are asked to provide details of any such tickets they hold and to identify public-transport journeys made using them during the seven days of the travel record. But attempting to distribute the cost of such a ticket across journeys made using it is not possible given that the ticket's validity may be much greater than the seven days during which travel is recorded. We therefore excluded public-transport trips made using a season ticket.

- We omitted long-distance journeys (categorised in the NTS as 50 miles or more).

- Whilst the National Travel Survey gathers information about vehicles held by the household and respondents are asked to identify which vehicle is used for a given journey made by private motorised transport, there was not sufficient resource to estimate operating costs at a vehicle level. We therefore applied a blanket rate for vehicle operating costs per kilometre.

- We restricted our analysis of private motorised transport to car and light van, excluding motorcycle. Given how small a proportion of total vehicle distance is travelled by motorcycle, ${ }^{8}$ we believe this to have been a justifiable simplification.

- The nature of our data licence meant that we were not provided with information about the cost of parking.

\section{Discussion}

Of these simplifications, we believe those relating to season tickets and long-distance trips are likeliest to be significant. With respect to the first, there is evidence that season-ticket ownership is more prevalent in higher-income groups [27]. A season ticket offers a saving over the walk-up fare in most cases so the consequence of omitting these trips will be to exaggerate the absolute cost of travel to a greater extent for rich travellers than for poor.

With respect to long trips, whilst they may be a small proportion of all reported trips (somewhat less than four per cent of the set ${ }^{9}$ ), they are by definition longer than most trips captured in the general travel record, whose average distance in 2015 was 7.3 miles [30]. Given that overall distance travelled is positively correlated with income [1], it seems reasonable to assume that wealthier people will make more long-distance trips than those from poorer backgrounds. Without analysing the speed and cost characteristics of the long-distance journey data, it is not possible to say with confidence what their omission means for this analysis. But it seems reasonable to assume that long-distance travel is both comparatively speedy and comparatively cheap per unit distance. We therefore suggest that the absence of these data from our analysis is likely to exaggerate the cost and time expended on travel and to do this to a greater extent in the case of wealthier individuals.

8 In 2015, approximately 2.8 billion miles were travelled by motorcycle in Great Britain. In comparison, 247.7 billion miles were travelled by cars and taxis, and 46.9 billion by light van [26].

9 There were 48,921 reported long-distance trips in the NTS across 2011 to 2015 [28]; over the same period, $1,377,572$ trips in all were recorded [29]. The matter is complicated by the fact that data on long-distance journeys are collected over two weeks in comparison with a single week for shorter trips. 
As can be seen, both discussions indicate that the simplifications made are likely to exaggerate the costs borne by wealthier travellers. Our findings are such that more comprehensive analysis would only strengthen the conclusions we are able to draw. 


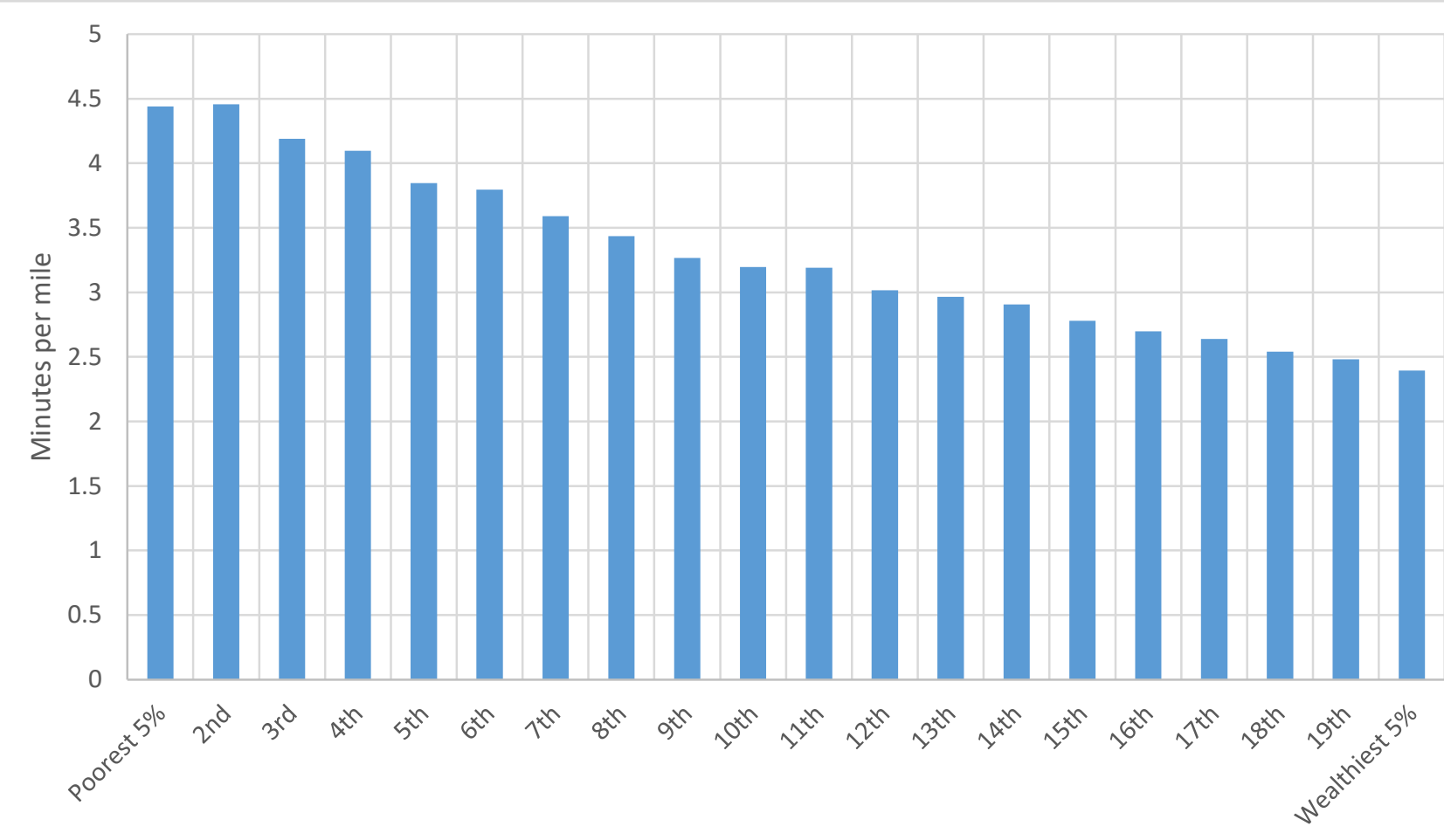

Household income semi-decile

Figure 1: Mean travel time per mile by income band

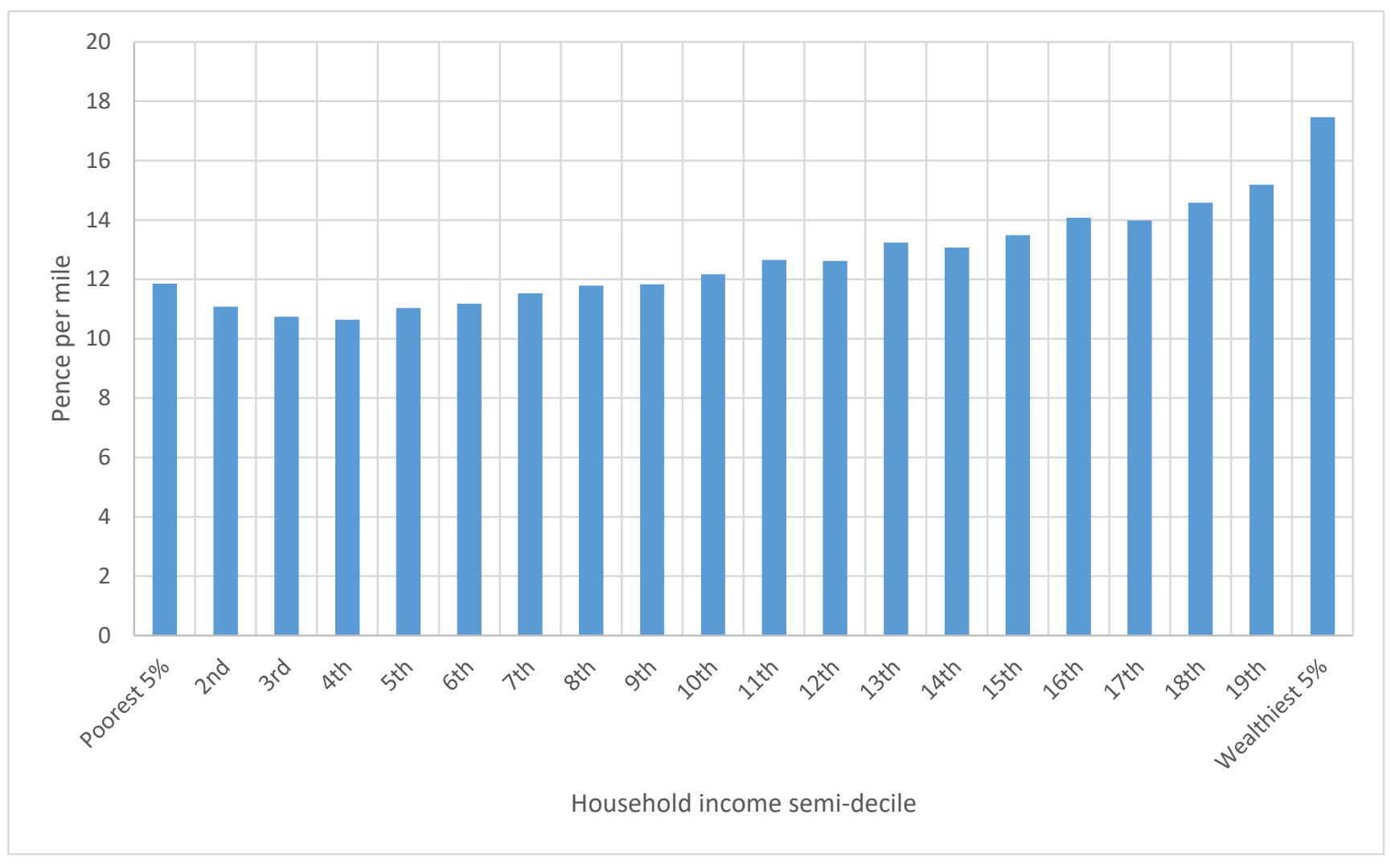

Figure 2: Mean cost of travel per mile by income band 


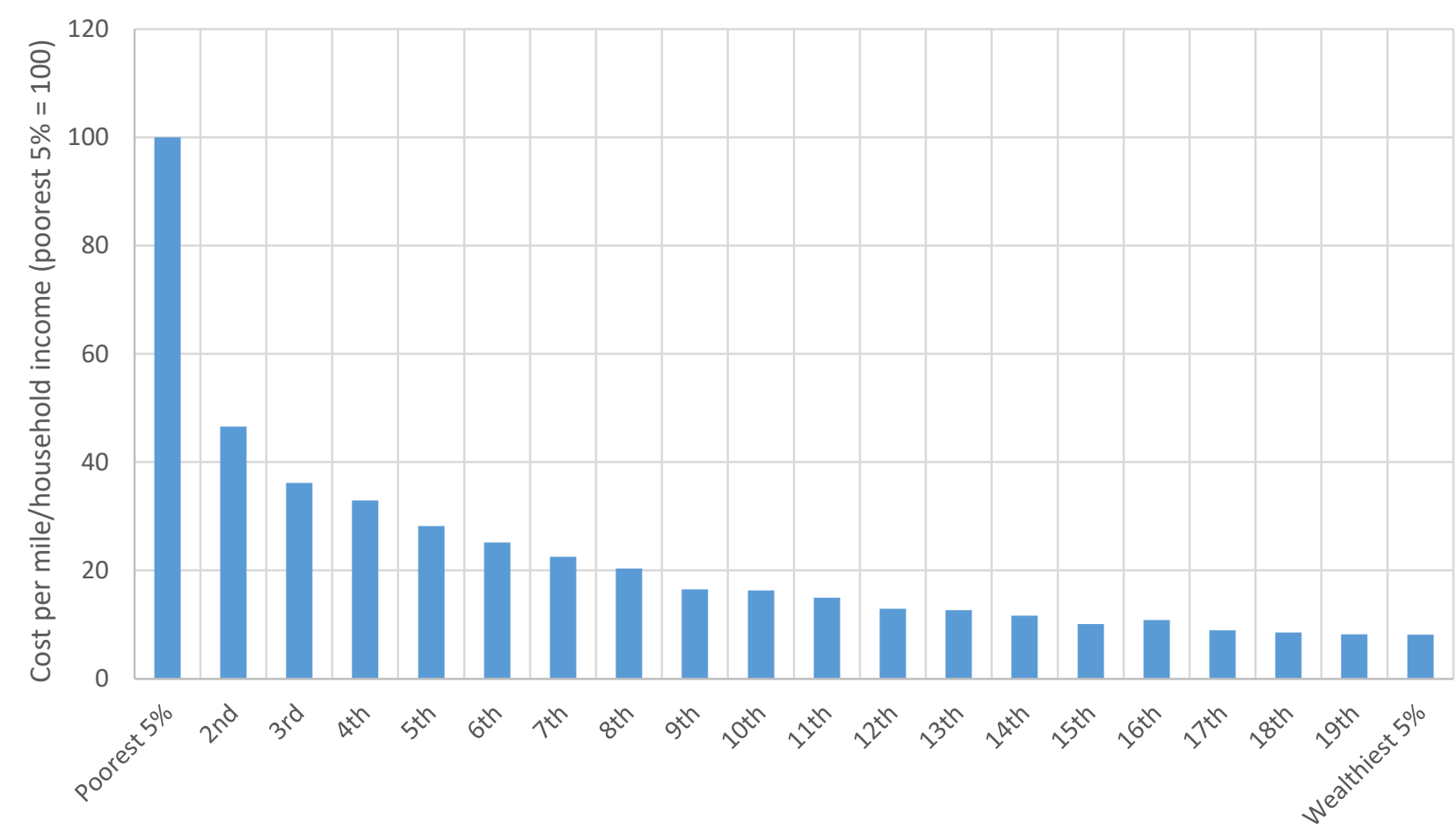

Household income semi-decile

Figure 3: Mean cost of travel per mile as proportion of household income, by income band 